\title{
EVALUATION OF NEUROGENIC CONTROL OF BLOOD PRESSURE IN HYPERTENSION WITH TETRAETHYLAMMONIUM CHLORIDE AND SPINAL ANESTHESIA ${ }^{1}$
}

\author{
By ALBERT A. BRUST,2 MORTON F. REISER, AND EUGENE B. FERRIS, JR. \\ (From the Departments of Internal Medicine and Psychiatry, University of Cincinnati College of \\ Medicine and the Cincinnati General Hospital, Cincinnati, Ohio)
}

(Submitted for publication September 25, 1950; accepted June 25, 1951)

The contribution of sympathetic constrictor impulses to arteriolar tone is now recognized as playing an important though sometimes variable role in the maintenance of increased peripheral resistance in some patients with hypertension. However, lack of a clear understanding of the exact nature of autonomic influences and of their importance in relation to other pressor factors has complicated the development and evaluation of therapeutic measures for hypertension which have as their aim the modification of the "neurogenic component." 3 Results obtained after surgical sympathectomy have seemed encouraging in certain patients, discouraging in others. It has become apparent that operative results are inconsistent and that the most striking inconsistency is the effect on the blood pressure. Difficult, too, is the choice of patients who may be expected to benefit from this type of treatment; no satisfactory method of selection has yet been suggested.

This study was undertaken in an effort to evaluate the nature of autonomic influences on blood pressure level as indicated by observations and comparisons of the responses to autonomic blockade with tetraethylammonium chloride ${ }^{4}$ (TEAC) and spinal anesthesia to high thoracic levels.

\section{MATERIAL AND METHODS}

Twenty-four patients were included in the study. Eleven patients had malignant and 13 had benign hyper-

1 The work described in this paper was aided in part by a grant from the Psychosomatic Research Fund of the National Committee for Mental Hygiene, Inc. and from the Life Insurance Medical Research Fund.

2 Research Fellow, American Heart Association.

3 As used here neurogenic has reference exclusively to mechanisms involving the functional integrity of the sympathetic nervous system. It is no longer tenable to consider psychogenic and neurogenic as synonymous terms since the utilization of humoral (non-neurogenic) pathways for emotional responses is now well-established.

* Etamon chloride supplied by Parke, Davis and Company, through the courtesy of Dr. E. C. Vonder Heide. tension. To determine the blood pressure effect of pharmacologic blockade of autonomic impulse transmission, TEAC in 3-5 cc. doses (300-500 mg.) was administered intravenously to all patients on from two to 12 occasions. All studies were conducted with the patients supine, and the injections were made rapidly, requiring no more than five seconds for administration. Observations of pupillary dilatation and abolition of sweating were accepted as clinical confirmation that autonomic blockade had been induced. In addition, in those patients who showed slight or moderate blood pressure depression following the drug, abolition of cold pressor reactivity was demonstrated to confirm autonomic block. Blood pressures were obtained by auscultation. The control blood pressure represents the mean of five or more measurements taken at oneminute intervals before TEAC administration and following stabilization of the pressure in the recumbent position. The lowest point to which the blood pressure descended in the first five minutes following injection has been termed the $T E A C$ floor, and for the purposes of this study this has been accepted as indicating that portion of arteriolar tone which persists following the removal of active autonomic influence. Although multiple tests with TEAC were done on all patients, Table I includes only the results of the first test. The first experience with TEAC was selected since comparison was to be made with a single first experience with spinal anesthesia.

On a subsequent day the effect on the blood pressure of spinal anesthesia to high thoracic levels was determined in each of these patients. With the subject on a tilt table, anesthesia was induced by the intrathecal injection of $100-125 \mathrm{mg}$. of procaine hydrochloride dissolved in 2-21/2 cc. of his own spinal fluid. Control blood pressures were obtained prior to anesthesia by the method described above. Immediately following the intrathecal injection, the patient was tilted 7-10 degrees head down, until the level of skin anesthesia to pin prick had risen to high thoracic levels. The patient was then returned to the horizontal position for the remainder of the study. In the majority of patients it was possible to confirm the anesthesia level to pin prick by the observation of well demarcated levels of sudomotor paralysis. Blood pressure readings were taken at minute intervals throughout the period of high anesthesia and were followed less frequently only after the level had receded below the thoracic segments. The mean of five consecutive blood pressure determinations during the period of the highest anesthesia level was taken as the spinal blood pressure floor. To- 
TABLE I

\begin{tabular}{|c|c|c|c|c|c|c|c|c|c|}
\hline \multirow{2}{*}{ Patient } & \multirow{2}{*}{ Diagnosis } & \multicolumn{2}{|c|}{ First TEAC } & \multicolumn{3}{|c|}{ Spinal anesthesia } & \multicolumn{3}{|c|}{ Spinal and TEAC } \\
\hline & & $\begin{array}{l}\text { Control } \\
\text { B.P. }\end{array}$ & $\begin{array}{l}\text { TEAC } \\
\text { B.P. floor }\end{array}$ & $\begin{array}{l}\text { Control } \\
\text { B.P. }\end{array}$ & $\begin{array}{l}\text { Spinal } \\
\text { B.P. floor }\end{array}$ & $\begin{array}{l}\text { Anes. } \\
\text { level }\end{array}$ & $\begin{array}{l}\text { Anes. } \\
\text { level }\end{array}$ & $\begin{array}{l}\text { Control } \\
\text { B.P. }\end{array}$ & $\begin{array}{c}\text { Spinal-TEAC } \\
\text { B.P. floor }\end{array}$ \\
\hline $\begin{array}{l}\text { I. O. } \\
\text { H. B. } \\
\text { J.W. } \\
\text { E. E. } \\
\text { F.I. } \\
\text { S. B. } \\
\text { L.S. } \\
\text { F. N. } \\
\text { M. Ma. } \\
\text { E. O. } \\
\text { D. H. } \\
\text { M. D. } \\
\text { O. H. } \\
\text { N. N. } \\
\text { P.T. } \\
\text { V. E. } \\
\text { E. H. } \\
\text { T. M. } \\
\text { H. L. } \\
\text { L. L. } \\
\text { M. Mo. } \\
\text { V. F. } \\
\text { D. Z. } \\
\text { E. B. }\end{array}$ & $\begin{array}{l}\text { M.H. } \\
\text { M.H. } \\
\text { B.H. } \\
\text { B.H. } \\
\text { M.H. } \\
\text { M.H. } \\
\text { B.H. } \\
\text { M.H. } \\
\text { B.H. } \\
\text { B.H. } \\
\text { M.H. } \\
\text { B.H. } \\
\text { B.H. } \\
\text { M.H. } \\
\text { M.H. } \\
\text { B.H. } \\
\text { B.H. } \\
\text { M.H. } \\
\text { M.H. } \\
\text { B.H. } \\
\text { B.H. } \\
\text { M.H. } \\
\text { B.H. } \\
\text { B.H. }\end{array}$ & $\begin{array}{l}252 / 174 \\
207 / 126 \\
230 / 150 \\
225 / 140 \\
218 / 148 \\
228 / 140 \\
181 / 142 \\
209 / 138 \\
224 / 154 \\
225 / 128 \\
217 / 151 \\
195 / 145 \\
218 / 145 \\
230 / 130 \\
199 / 144 \\
182 / 116 \\
220 / 135 \\
240 / 134 \\
225 / 132 \\
188 / 98 \\
170 / 95 \\
196 / 154 \\
150 / 103 \\
199 / 120\end{array}$ & $\begin{array}{l}180 / 120 \\
134 / 86 \\
170 / 130 \\
138 / 96 \\
120 / 90 \\
210 / 126 \\
160 / 130 \\
105 / 80 \\
140 / 100 \\
176 / 110 \\
170 / 110 \\
105 / 90 \\
185 / 130 \\
162 / 100 \\
142 / 104 \\
114 / 76 \\
160 / 105 \\
176 / 110 \\
174 / 110 \\
150 / 80 \\
140 / 75 \\
114 / 84 \\
135 / 95 \\
134 / 86\end{array}$ & $\begin{array}{l}258 / 162 \\
240 / 146 \\
209 / 146 \\
236 / 153 \\
240 / 155 \\
262 / 160 \\
198 / 155 \\
260 / 160 \\
239 / 153 \\
253 / 137 \\
230 / 153 \\
240 / 158 \\
210 / 151 \\
222 / 139 \\
256 / 163 \\
200 / 118 \\
210 / 130 \\
219 / 138 \\
253 / 175 \\
184 / 101 \\
225 / 125 \\
215 / 154 \\
170 / 122 \\
204 / 123\end{array}$ & $\begin{array}{l}167 / 106 \\
172 / 99 \\
132 / 96 \\
173 / 112 \\
184 / 125 \\
219 / 135 \\
160 / 130 \\
146 / 98 \\
190 / 128 \\
143 / 95 \\
140 / 91 \\
200 / 138 \\
188 / 130 \\
194 / 116 \\
198 / 132 \\
146 / 89 \\
164 / 108 \\
185 / 110 \\
171 / 113 \\
162 / 95 \\
150 / 94 \\
127 / 102 \\
141 / 107 \\
180 / 119\end{array}$ & $\begin{array}{l}T_{5-6} \\
T_{4-5} \\
T_{2} \\
T_{4} \\
T_{4} \\
T_{5-6} \\
T_{4-5} \\
T_{4-5} \\
T_{2} \\
T_{4-5} \\
T_{2-3} \\
T_{6} \\
T_{2-4} \\
T_{6} \\
T_{5-6} \\
T_{4-6} \\
T_{4-5} \\
T_{2-4} \\
T_{4} \\
T_{2} \\
T_{2-4} \\
T_{4} \\
T_{3-4} \\
T_{4-6}\end{array}$ & $\begin{array}{l}T_{8} \\
T_{7-8} \\
T_{0} \\
T_{6-7} \\
T_{7-8} \\
T_{6-7} \\
T_{8} \\
T_{8} \\
T_{3} \\
T_{7-8} \\
T_{3-1} \\
T_{6-7} \\
T_{6-7} \\
T_{6-7} \\
T_{7-8} \\
T_{7} \\
T_{9} \\
T_{7} \\
= \\
= \\
= \\
=\end{array}$ & $\begin{array}{l}182 / 108 \\
186 / 100 \\
160 / 110 \\
170 / 114 \\
205 / 135 \\
246 / 144 \\
160 / 130 \\
200 / 120 \\
190 / 128 \\
155 / 105 \\
140 / 91 \\
190 / 140 \\
205 / 135 \\
206 / 120 \\
204 / 140 \\
178 / 100 \\
155 / 100 \\
188 / 108 \\
= \\
= \\
= \\
=\end{array}$ & $\begin{array}{c}156 / 100 \\
140 / 90 \\
150 / 110 \\
116 / 84 \\
170 / 115 \\
198 / 128 \\
150 / 110 \\
75 / 65 \\
154 / 118 \\
150 / 105 \\
135 / 90 \\
145 / 125 \\
150 / 120 \\
136 / 90 \\
220140 \\
112 / 70 \\
125 / 85 \\
184 / 110 \\
= \\
= \\
= \\
=\end{array}$ \\
\hline
\end{tabular}

ward the end of the experiment, usually after the upper anesthesia level had receded to the lower thoracic segments, and the blood pressure had begun to rise, the TEAC floor was again determined in 18 of the 24 cases.

\section{RESULTS}

The results are summarized in Table I. In all patients, the blood pressure was reduced significantly by both TEAC and spinal anesthesia whether administered separately or in combination. There was a clear parallelism between the degree of blood pressure fall to the two procedures, and the blood pressure floors achieved by autonomic blockade with TEAC were slightly but significantly lower than the floors obtained with spinal anesthesia to levels ranging from $T_{6}$ to $T_{3}$.

Despite individual differences in magnitude of the depressor response at similar levels of spinal anesthesia, it was noted in all cases that the greatest reduction in blood pressure occurred when the anesthesia level was highest. As anesthesia receded from the higher spinal segments the blood pressure ascended in parallel fashion. There was, however, much individual variation in the rate of blood pressure recovery to control levels and such levels were rarely achieved during the period of observation even though evidences of anesthesia had disappeared.
It was repeatedly noted that control blood pressure levels were consistently higher than usual just prior to induction of spinal anesthesia. This seemed to correlate with other evidence of anticipatory anxiety which was observed to diminish as the experiment proceeded and may explain the failure of the blood pressure to return to preanesthetic levels in many cases. There was a similar but less marked tendency for the control blood pressure to be higher than usual before the first test with TEAC, and subsequent TEAC testing revealed a general trend to slightly lower control blood pressures when the experience was repeated.

In only four of the 24 patients studied was a lower blood pressure level obtained with high spinal anesthesia than with the TEAC test performed previously. Comparison of the differences between the spinal anesthesia floor and the first TEAC floor shows that the TEAC floor is significantly lower for this group of patients (systolic, $p$ less than 0.02 ; diastolic, $p$ less than 0.01$).^{5}$

5 Because of space limitations only data relating to the first TEAC test are reported. However, analysis of the difference between the spinal floor and the closest temporally related TEAC floor, and the means of all TEAC floors showed likewise a high degree of statistical significance. 
Spinal anesthesia lowered the blood pressure from a mean control level of $226 / 145 \mathrm{~mm}$. $\mathrm{Hg}$ to $168 / 111 \mathrm{~mm}$. Hg. Mean control blood pressure before TEAC was $210 / 131 \mathrm{~mm}$. $\mathrm{Hg}$, and the mean TEAC floor was $150 / 101 \mathrm{~mm}$. Hg.

In 12 patients the upper level of anesthesia achieved was between $T_{6}$ and $T_{4}$. For this group the mean difference between spinal floor and first TEAC floor was $27.2 / 18.7 \mathrm{~mm}$. Hg. In the remaining 12 patients anesthesia height was from $\mathrm{T}_{4}$ to $\mathrm{T}_{2}$ and the mean floor difference $10.9 / 7.8$ $\mathrm{mm}$. Hg. Thus, although specific patient to pa-
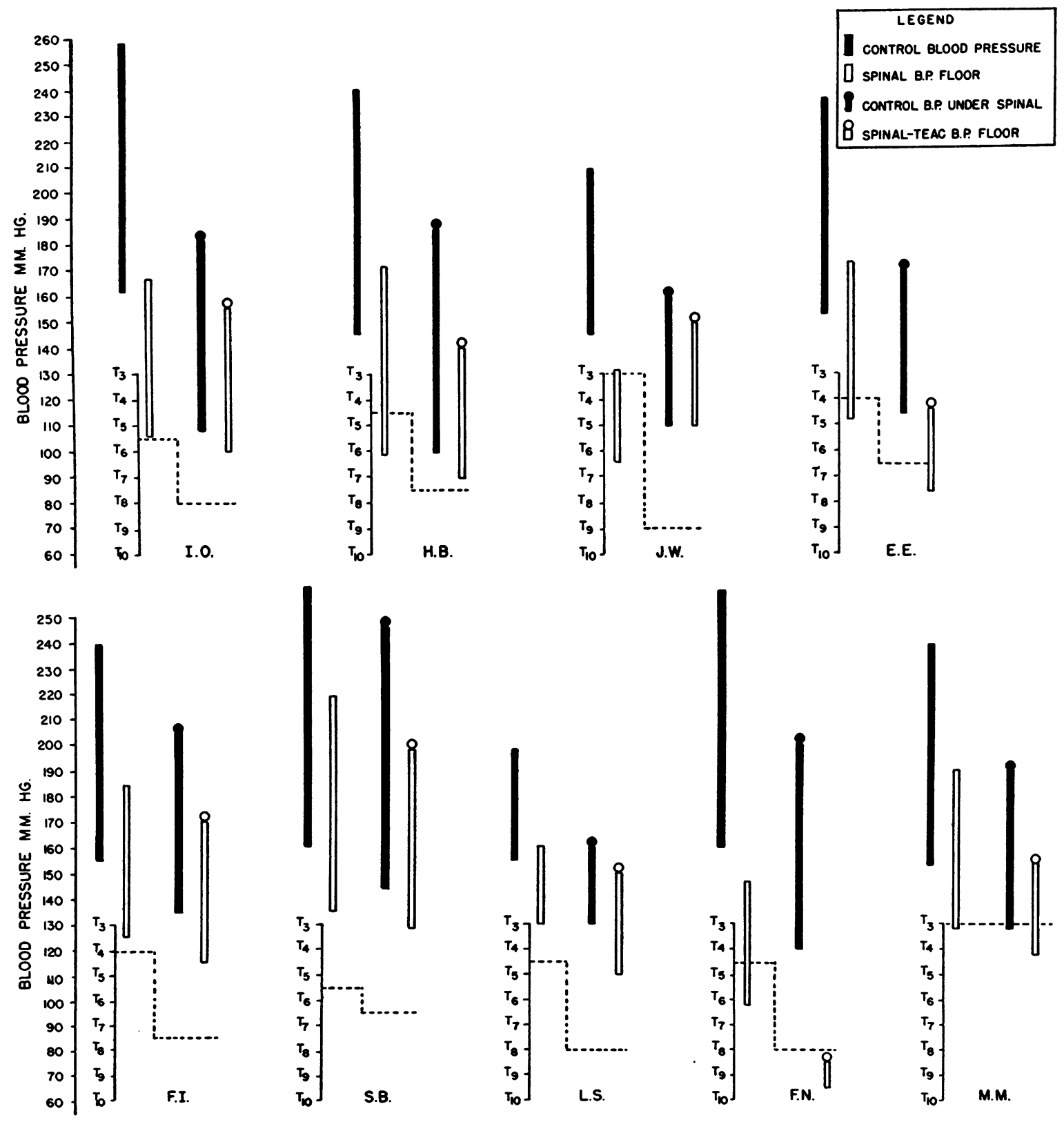

Fig. 1. Comparison of Spinal and Spinal-TeaC Blood Pressure Floors

The columns as indicated in the legend represent blood pressure levels which may be read from the scale at the extreme left. The dotted line extending from the spinal segment scale indicates the upper level of spinal anesthesia at the time of the blood pressure observations. 
tient correlations of anesthesia level and blood pressure level are not feasible, there is a definite trend for the spinal anesthesia floor to approach the TEAC floor as the level of anesthesia is carried higher. These observations have served to focus attention on the upper thoracic segments as mediating a significant portion of the sympathetic constrictor tone in these patients.
In 18 patients the TEAC floor was again determined during the period of spinal anesthesia, usually after anesthesia had begun to recede from the higher thoracic segments. Individual results are shown in Figures 1 and 2. The mean TEAC floor under spinal $(148 / 103 \mathrm{~mm} . \mathrm{Hg})$ was $25 / 10$ $\mathrm{mm}$. $\mathrm{Hg}$ lower than the spinal floor $(173 / 113 \mathrm{~mm}$. $\mathrm{Hg}$ ) and was almost identical with the first TEAC
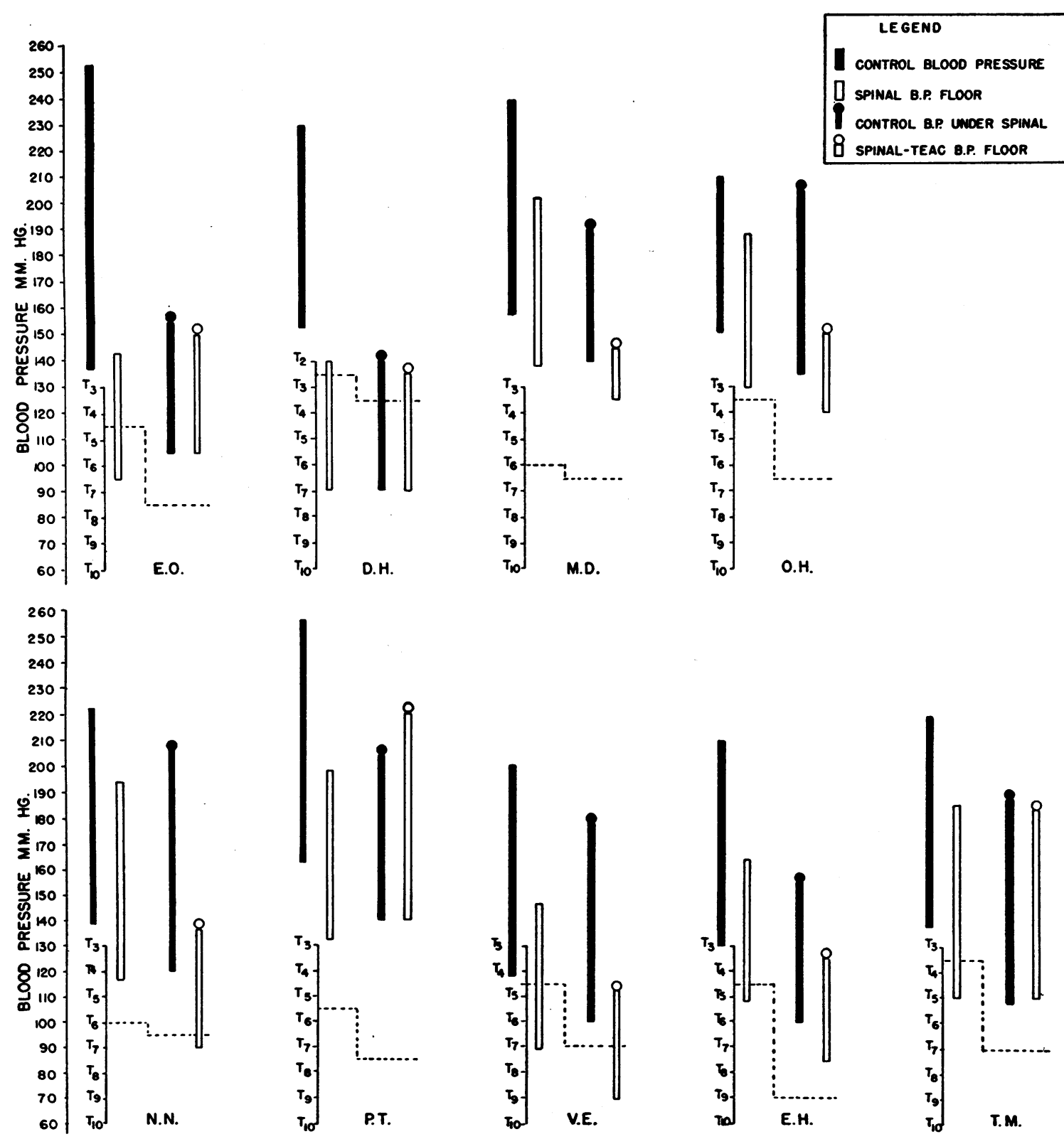

Fig. 2. Comparison of Spinal and Spinal-TEAC Blood Pressure Floors For explanation see Figure 1 . 


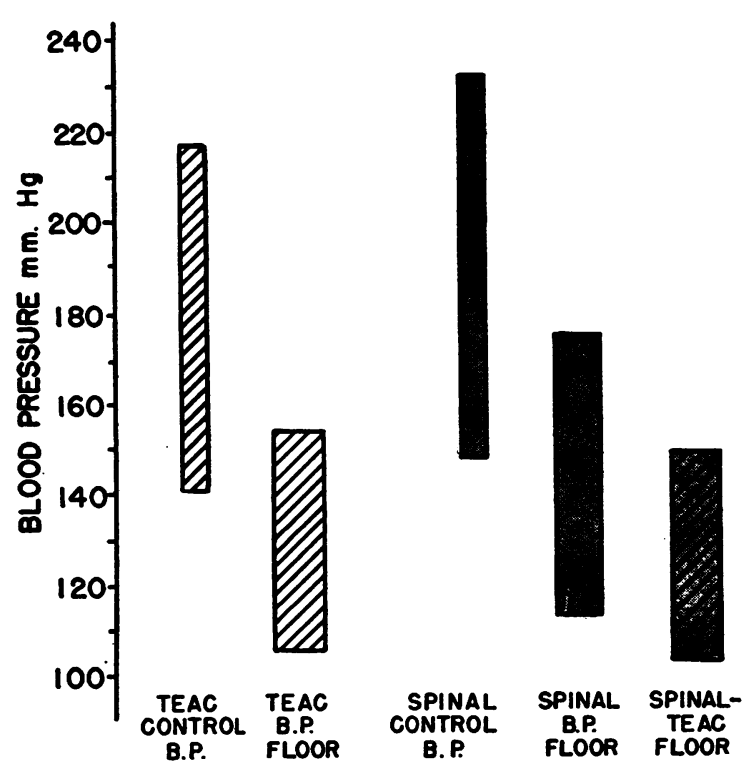

Fig. 3. Comparison of Means

Mean blood pressure responses of 18 patients who, following an initial test with TEAC, received the same drug during receding high spinal anesthesia on a subsequent day. Note the similarity of the initial mean TEAC blood pressure floor to that obtained during anesthesia.

floor $(153 / 105 \mathrm{~mm} . \mathrm{Hg})$ for these same patients (Figure 3). Statistical evaluation of the differences between the spinal floor and the TEAC floor under receding spinal anesthesia reveals a high degree of significance (systolic and diastolic, $p$ less than 0.01 ) whereas comparison of the differences between the first TEAC floors and the TEAC floors under receding spinal anesthesia shows no statistical significance (systolic, $p$ greater than 0.5 ; diastolic, $p$ greater than 0.7 ).

\section{DISCUSSION}

The blockade of autonomic ganglia induced by TEAC and the pre-ganglionic block achieved by high spinal anesthesia exhibit certain hemodynamic similarities. It is generally accepted that the arteriolar dilatation which is observed with both procedures occurs as a result of the interruption of tonic vasomotor impulses and reflects a diminution in peripheral resistance. Cardiac output is not reduced by TEAC (1) and need not change after spinal anesthesia. Harrison (2) emphasizes that when cardiac output does fall during spinal anesthesia, the fall occurs secondarily, i.e., follows rather than precedes any reduction in blood pressure. Studies by Milwidsky and de Vries on intramuscular pressures (3) and by Sarnoff and Arrowood with differential spinal block (4) have offered added support to the concept that sympathetic constrictor paralysis is the principal mechanism involved in the reduction of blood pressure during spinal anesthesia.

Questions as to the completeness of the ganglionic block by TEAC have been raised. Evidence presented here and elsewhere (5) indicates that for the purpose of blood pressure evaluation in acute intravenous experiments, the block is sufficiently complete although of rapidly transient nature.

Thus in this study of patients with hypertension an attempt has been made to compare blood pressure levels after relatively complete sympathetic block (TEAC) with those obtained during an anatomically less complete sympathetic paralysis (high spinal anesthesia). The results demonstrate clearly that in the majority of cases studied, the blood pressure falls to lower levels in response to TEAC block than during high spinal anesthesia regardless of whether the TEAC is given alone or during receding anesthesia. A further indication of more complete block by TEAC is seen in the tendency for the spinal blood pressure floor to approach the TEAC floor as the anesthesia level is carried higher.

Maximum blood pressure reduction at the highest anesthesia level and progressive blood pressure rise as the anesthesia level recedes are consistent with the observations of Russek, Southworth and Zohman during continuous caudal anesthesia (6). It thus appears that in these cases of hypertension there is a direct relationship between the level of the blood pressure and the extent of the arteriolar bed under active vasomotor control. All of these observations substantiate a 1932 report by Ferguson and North (7) that the amount of blood pressure fall which occurs with spinal anesthesia bears a direct relationship to the number of white rami anesthetized.

Since the degree of additional fall in blood pressure induced by TEAC varies widely in different patients with spinal anesthesia of comparable extent in the current study, it appears that the remaining portion of the arteriolar bed (unanes- 
thetized portion) which is capable of exerting pressor activity varies from patient to patient. This observation may help to explain the lack of uniform blood pressure responses when sympathetic resections of standardized extent are performed on large groups of patients. It is of interest that in a parallel study of the cold pressor test (8), it was found that neurogenic control of cold pressor reactivity follows the same pattern as seen here for the neurogenic vasomotor mechanism.

Taylor and associates (9) were unable to derive other than negative value from pre-sympathectomy spinal and caudal anesthesia studies in hypertensive patients. Soloff and coworkers (10), using tetraethylammonium bromide and spinal anesthesia likewise found that they could not consistently predict post-operative blood pressure levels. The TEAC floor has been interpreted here to reflect that component of arteriolar tone which is relatively devoid of sympathetic control. It has previously been reported that in some patients this floor may vary considerably from day to day (11), and hence that such variation may represent fluctuation of interacting neurogenic and humoral (non-neurogenic) mechanisms. Some of the patients in this study who had repeated TEAC tests likewise showed this variability. Whether similar and parallel fluctuations in the response to spinal anesthesia occur in these same patients was not determined here. Current evidence for day to day variability of the neurogenic component in many hypertensive patients may help account for the apparent discrepancies in pre-operative predictions and post-operative observations. Repeated pre-operative study of the blood pressure effects of sympathetic block in a given patient may offer a clue to the degree of variability of the neurogenic component in the individual case. Inconsistent post-operative blood pressure results thereby become more meaningful when pre-operative variability to autonomic block has been established. In our experience, repeated testing with TEAC may be required to assess the degree of consistency or variability of response. It should be remembered that the results of this study represent the immediate effects of acute disruption of the homeostatic mechanisms involved in blood pressure maintenance. It need not necessarily follow that the long term effects of more permanent blocking procedures will parallel these acute phenomena. However, in patients who show a significant neurogenic component (as indicated by sizeable and reproducible blood pressure reduction in response to spinal anesthesia and/or TEAC) and for whom, on the basis of clinical appraisal, sympathectomy seems advisable, it would appear reasonable to test this hypothesis by varying the extent of operation according to the result of such testing for each individual case.

\section{CONCLUSIONS}

1. In these patients with hypertension a clear parallelism has been demonstrated between the degree of blood pressure fall to TEAC and to that induced by high spinal anesthesia.

2. The fact that in these patients lower blood pressure levels were obtained with TEAC than with high spinal anesthesia, plus the ability of TEAC to reduce the blood pressure further when administered during high spinal anesthesia, provide additional evidence that the pharmacologic autonomic blockade which this drug induces is relatively more complete than that which occurs with the block of extensive but anatomically incomplete spinal anesthesia.

3. In these patients the level of the blood pressure tended to vary indirectly with the level of spinal anesthesia.

\section{ACKNOWLEDGMENTS}

The authors wish to express their appreciation to Miss Mary A. Costello, chief anesthetist of the Department of Surgery, University of Cincinnati, and to her assistants for their aid in administering the spinal anesthesia. The contributions and assistance of Drs. Alvin P. Shapiro, Harrison M. Baker, Joseph Levinson and Mrs. Jane K. Friedlander are also gratefully acknowledged.

\section{REFERENCES}

1. Lyons, R. H., Hoobler, S. W., Neligh, R. B., Moe, G. K., and Peet, M. M., Experiences with tetraethylammonium chloride in hypertension. J.A.M.A., 1948, 136, 608.

2. Harrison, T. R., Failure of the Circulation. Baillière, Tindall \& Cox, London, 1939, 2nd ed.

3. Milwidsky, H., and de Vries, A., Regulation of blood pressure during spinal anesthesia: observations on intramuscular pressure and skin temperature. Anesthesiology, 1948, 9, 258. 
4. Sarnoff, S. J., and Arrowood, J. G., Differential spinal block. II. The reaction of sudomotor and vasomotor fibers. J. Clin. Invest., 1947, 26, 203.

5. Ferris, E. B., Reiser, M. F., Stead, W. W., and Brust, A. A., Jr., Clinical and physiological observations of interrelated mechanisms in arterial hypertension. Tr. A. Am. Physicians, 1948, 61, 97.

6. Russek, H. I., Southworth, J. L., and Zohman, B. L., Selection of hypertensive patients for sympathectomy. J.A.M.A., 1946, 130, 927.

7. Ferguson, L. K., and North, J. P., Observations on experimental spinal anesthesia. Surg., Gynec. \& Obst., 1932, 54, 621.

8. Reiser, M. F., and Ferris, E. B., The nature of the cold pressor test and its significance in relation to neurogenic and humoral mechanisms in hypertension. J. Clin. Invest., 1948, 27, 156.

9. Taylor, R. D., Birchall, R., Corcoran, A. C., and Page, I. H., Circulatory responses to spinal and caudal anesthesia in hypertension: relation to the effect of sympathectomy. I. Effect on arterial pressure. Am. Heart J., 1948, 36, 221.

10. Soloff, L. A., Burnett, W. E., and Bello, C. T., A study of the comparative value of tetraethylammonium bromide and spinal anesthesia in selection of hypertensive persons for sympathectomy. Am. J. M. Sc., 1948, 216, 665.

11. Levinson, J. E., Reiser, M. F., and Ferris, E. B., Variations in the blood pressure response to repeated administration of tetraethyl ammonium chloride. J. Clin. Invest., 1948, 27, 154. 